\title{
Collaboration is Key - Bridging the Gap and Building an IR Endoscopy Practice
}

Nathan R. Albertson, MD ${ }^{1}$; Jeffrey Forris Beecham Chick, MD, MPH, DABR ${ }^{2}$; Albert Jiao, BS ${ }^{3}$; Travis L. Healey, MD ${ }^{1}$; Adam N. Plotnik, MD ${ }^{4}$; Ravi N. Srinivasa, MD, FSIR ${ }^{4}$

${ }^{1}$ Department of Radiology and Imaging Sciences, Indiana University School of Medicine

${ }^{2}$ Division of Vascular and Interventional Radiology, Inova Alexandria Hospital, 4320 Seminary Road, Alexandria, VA 22304

${ }^{3}$ Michigan State University College of Human Medicine, Secchia Center, 15 Michigan Street N.E., Grand Rapids, MI 49503

${ }^{4}$ Department of Radiology, Division of Vascular and Interventional Radiology, UCLA Medical Center, 757 Westwood Plaza, Los Angeles, CA 90095

Abstract. While the tools and techniques employed by interventional radiologists on a day-to-day basis translate well to learning the skills required to perform basic endoscopic interventions, collaboration with other specialties is crucial to the success of an interventional radiology endoscopy program. As in any field in medicine, the paramount goal is to improve patient care. Adding the ability to directly visualize structures through an endoscope to certain interventional radiologic procedures may greatly augment the efficacy, safety, and success of interventional radiology procedures. Colleagues in urology, gastroenterology, and surgery should be involved in decision making and treatment planning to ensure that a shared vision for optimal patient care is achieved.

KEYWORDS: Collaboration; choledochoscopy; cholangioscopy; cholangiocystoscopy; stricture; cost

ABBREVIATIONS: IR â€“ interventional radiology; ERCP â€“" endoscopic retrograde cholangiopancreatography

This is the author's manuscript of the article published in final edited form as:

Albertson, N. R., Chick, J. F. B., Jiao, A., Healey, T. L., Plotnik, A. N., \& Srinivasa, R. N. (2019). Collaboration is Key - Bridging the Gap and Building an IR Endoscopy Practice. Techniques in Vascular and Interventional Radiology. https://doi.org/10.1053/j.tvir.2019.04.003 
In any field of medicine, providing quality, competent patient care is the overarching goal. In interventional radiology, we provide unique services to patients that can both save lives and improve quality of life. Within IR, endoscopy provides an opportunity to expand such services, but it cannot be performed without a multidisciplinary approach that brings together the patient's care team. Other specialties that perform endoscopy, as an integral part of their practice, may become defensive of their procedures. However, through working with these other specialists and offering unique forms of endoscopy, an interventional radiologist can demonstrate that endoscopy performed by IR does not threaten their practice and instead provides new treatments for patients who often have few other options.

There are multiple conflicts which may arise that providers must work through when adopting endoscopy. While interventional radiologists have leeway to make medical decisions intra-procedurally, explicitly working in opposition to a consulting provider's requests is inappropriate. If the provider requesting an IR procedure specifies 'do not,' it is often best to oblige, as the requesting colleague may have more intimate knowledge of the patient's history and preferences. Furthermore, if a provider did not know IR had viable treatment options for a patient, they would never have placed the initial consult. It is therefore important that our colleagues in other specialties know both what treatments we can offer and when they should be performed. Finally, they must trust that we can provide that treatment adequately. This is true across radiology but may provide a larger barrier to performing endoscopic procedures. Building endoscopy into an IR practice takes the same skills as being a doctor: dedication to learning, getting along with others, and a desire to help patients.

\section{AS AN ADJUNCT TO CURRENTIR PROCEDURES}

Some ways an interventional radiologist can incorporate endoscopy into his or her practice may purely enhance treatments their practice already offers. For example, in practices where interventional radiology places antegrade ureteral stents, endoscopy can assist in traversing strictures. Imagine a renal transplant patient, status post pyelovesicostomy, who has now developed a stricture. Multiple attempts to stent the stricture percutaneously have failed, as has a retrograde inserted double-J stent. Using percutaneous endoscopy in addition to fluoroscopy, radiologists can traverse the stricture under direct visualization and successfully place the stent. One could assume both the patient and the urologist would be appreciative of the radiologist's work [1].

\section{PROCEDURES UNIQUE TO IR}

Other endoscopic options may not just alter an IR procedure, but instead represent a new procedure that IR is most well-suited to perform. For example, percutaneous biliary access is often performed for imaging assessment or treatment of biliary strictures and stones. Even in these procedures, endoscopy may be of assistance. Some stones may be too large to force through the ampulla of Vater or the percutaneous tract. There are various options for fragmenting these stones, one of 
which is laser lithotripsy. This method is augmented by endoscopic visualization of the stone, which can even be performed alongside an endourologist operating the scope [26].

While choledochoscopy, cholangioscopy, and cholangiocystoscopy have been described to assist with stones and strictures, they can also be used to perform biopsy. The use of percutaneous cholangioscopy has been described for the characterization of cholangiocarcinoma instead of peroral cholangioscopy. This traditional endoscopic route allows for assessment of proximal tumor extent and the evaluation of intrahepatic lesions, which may also be possible with percutaneous assessment [5-7]. Only in discussions with oncology, gastroenterology, and surgery can a patient's best option be determined.

\section{AVOIDING SURGERY}

In patients who may benefit from peroral cholangioscopy, not everyone can easily undergo the procedure. In patients with complex, surgically altered anatomy, traditional treatment modalities may not be available. These patients include those who have undergone Roux-en-Y gastric bypass, duodenal switch, or other anatomy-altering surgeries. For these patients to have an ERCP, they must either undergo a complex, balloon assisted ERCP, or surgically assisted ERCP [8]. By offering a percutaneous option for imaging (cholangiogram), treatment (for stones and strictures), and diagnosis (biopsy), interventional radiologists allow patients to avoid surgery and endoscopists to save time that they can otherwise dedicate to more efficient patient care.

\section{COST SAVINGS}

Some of the endoscopes used are disposable, but others are reusable. By utilizing reusable endoscopes, the cost-per-use is reduced, saving money for patients and the healthcare system. If these endoscopes can be shared among specialties, then more proceduralists are able to reap the benefits. Even when disposable scopes are used, the equipment required to use them can cost thousands of dollars. Distributing the costs of the light sources, cameras, monitors, and other equipment can provide benefit to the patient and providers. The radiologist must work with administrators and other endoscopists to both determine the best equipment options and obtain said equipment.

Clearly, when properly used, interventional radiology operated endoscopy can provide patients with quality, cost-effective care they may not otherwise receive. To offer this care, interventional radiologists must work closely with providers in other specialties. Some physicians may demonstrate these capabilities in formal multidisciplinary presentations. Another way to recruit appropriate patients may be in case conferences, or even in informal conversations. Regardless, working with other specialties has always been an important part of radiology, and endoscopy is no different. 


\section{REFERENCES}

1. Chick, J.F.B., et al., Disposable Single-Use Ureteroscopy-Guided Nephroureteral Stent Placement in a Patient with Pyelovesicostomy Stricture and Failed Prior Nephroureteral Stent Placement. J Vasc Interv Radiol, 2017. 28(9): p. 13191321.

2. Collins, D., et al., Biliary endoscopy: an opportunity for interventional radiology. Journal of Vascular and Interventional Radiology, 2015. 26(2): p. S193-S194.

3. Copelan, A. and B.S. Kapoor, Choledocholithiasis: Diagnosis and Management. Tech Vasc Interv Radiol, 2015. 18(4): p. 244-55.

4. Patel, N., et al., Interventional radiology operated endoscopy using the LithoVue disposable endoscope: approach, technical success, complications, and outcomes in 12 patients. Journal of Vascular and Interventional Radiology, 2018. 29(4): p. S243-S244.

5. Schlachter, T., et al., Percutaneous transhepatic cholangioscopy: outcome as a diagnostic and therapeutic tool in the management of biliary tract disease. Journal of Vascular and Interventional Radiology, 2014. 25(3): p. S50-S51.

6. Tower, S.W. and J.A. Requarth, Evaluation of a newly instituted percutaneous antegrade choledochoscopy program for biliary disease in poor surgical candidates. Journal of Vascular and Interventional Radiology, 2014. 25(3): p. S50.

7. Nimura, Y., Staging cholangiocarcinoma by cholangioscopy. HPB (Oxford), 2008. 10(2): p. 113-5.

8. Ross, A.S., Techniques for Performing ERCP in Roux-en-Y Gastric Bypass Patients. Gastroenterol Hepatol (N Y), 2012. 8(6): p. 390-2. 